\title{
Effectiveness and Costs Among Rheumatoid Arthritis Patients Treated with Targeted Immunomodulators Using Real-World U.S. Data
}

\author{
Mahdi Gharaibeh, PharmD, MSc, PhD; Machaon Bonafede, PhD, MPH; Donna McMorrow, BS; \\ Ervant J. Maksabedian Hernandez, PhD, MPhil; and Bradley S. Stolshek, PharmD
}

\begin{abstract}
BACKGROUND: Targeted immunomodulators (TIMs) are used for the treatment of moderate to severe rheumatoid arthritis (RA) and include biologic and nonbiologic medications with different mechanisms of action. Data describing disease activity levels in RA are not directly available in claims databases but can be determined using a claims-based effectiveness algorithm. Rheumatology has benefited from the recent introduction of new drugs, many with new mechanisms of action. We provide an analysis of this broader range of medications.
\end{abstract}

OBJECTIVES: To (a) describe and summarize the effectiveness of available TIMs for the treatment of moderate to severe RA and (b) determine the RA-related health care costs per effectively treated patient, using recent data.

METHODS: This was a retrospective analysis using data from the IBM MarketScan Commercial Claims and Encounters Database from July 1, 2012, through December 31, 2016. Index date was the new prescription claim for a TIM (abatacept, adalimumab, certolizumab pegol, etanercept, golimumab, infliximab, tocilizumab, or tofacitinib). A 6-month pre-index baseline period was used to determine demographic and clinical characteristics. Patients without a TIM claim during the baseline period were considered naive; patients with a TIM claim in the baseline period that was different than the index TIM were assessed as receiving second-line therapy. A claims-based algorithm was used to assess 12-month treatment effectiveness and total RA-related costs. Costs included RA-related pharmacy costs and medical costs.

RESULTS: Data from 14,775 patients were analyzed, including patients prescribed abatacept $(n=1,250)$, adalimumab $(n=4,986)$, certolizumab pegol $(n=387)$, etanercept $(n=5,266)$, golimumab $(n=577)$, infliximab ( $n=969)$, tocilizumab $(n=451)$, and tofacitinib $(n=889)$. Of these, 705 were receiving second-line therapy. TIM effectiveness by first-line and second-line therapy, respectively, were abatacept $27.1 \%, 18.1 \%$; adalimumab $30.9 \%$, $22.1 \%$; certolizumab pegol $20.9 \%$, $14.3 \%$; etanercept $31.4 \%, 31.5 \%$; golimumab $32.7 \%$, 22.2\%; infliximab $21.9 \%, 21.3 \%$; tocilizumab $30.9 \%, 30.6 \%$; and tofacitinib $26.0 \%, 21.6 \%$. The main reason for failing effectiveness was not achieving an $80 \%$ medication possession ratio or being nonadherent. The 1-year total RA-related cost per effectively treated patient for first-line and second-line therapies, respectively, were abatacept $\$ 121,835, \$ 174,090$; adalimumab $\$ 112,708, \$ 154,540$; certolizumab pegol \$149,946, \$236,743; etanercept \$102,058, \$94,821; golimumab $\$ 108,802, \$ 140,651$; infliximab $\$ 155,123, \$ 185,369$; tocilizumab $\$ 93,333, \$ 109,351$; and tofacitinib $\$ 100,306, \$ 130,501$.

CONCLUSIONS: The effectiveness of TIMs from this real-world experience showed that the range of patients who were effectively treated with first-line therapy was higher for certain tumor necrosis factor inhibitors and tocilizumab. The percentages of effectively treated patients were generally lower in second-line treatment compared with first-line except for etanercept, which had the same percentage between lines of therapy. Etanercept had the lowest RA-related cost per effectively treated patient among tumor necrosis factor inhibitors in first-line use and the lowest
RA-related cost per effectively treated patient compared with all secondline treatments.

J Manag Care Spec Pharm. 2020;26(8):1039-49

Copyright $\odot 2020$, Academy of Managed Care Pharmacy. All rights reserved.

\section{What is already known about this subject}

Targeted immunomodulators (TIMs) are effective at reducing the signs and symptoms, improving health-related quality of life, and halting radiographic progression of joint disease in patients with moderate to severe rheumatoid arthritis (RA); however, patients have variable responses to these medications.

A validated, claims-based algorithm has been used to identify these effectively treated patients in a claims database.

\section{What this study adds}

Despite more options and opportunities for switching therapy, first-line treatment effectiveness rates for existing TIMs were similar to those observed in prevous studies, while newer TIMs were slightly less effective in first-line and second-line use.

The percentages of effectively treated patients varied, except for etanercept and infliximab, and were generally lower for secondline therapy than for first-line therapy.

The mean costs of 1-year RA-related costs per effectively treated patient varied, and, except for etanercept, were higher for second-

line therapy than for first-line therapy.
$\mathrm{R}$ heumatoid arthritis (RA) affects approximately 41 in 100,000 people in the United States, representing about 1.5 million adults. ${ }^{1}$ The high prevalence and long-term disability associated with RA have a significant effect on health care systems. ${ }^{2}$ Direct costs, which are managed by payers, include medications, diagnostic procedures and tests, hospitalizations, professional fees, therapeutic interventions, mechanical aids, physiotherapy, and nursing expenses. ${ }^{3}$ In 2003, the direct cost of RA and other rheumatic conditions was $\$ 80.8$ billion in the United States, and indirect costs were $\$ 47$ billion. $^{4}$ Effective treatment for RA is necessary to prevent or reduce direct and indirect costs.

Health care claims are a common source of real-world data to evaluate health care resource utilization and costs; however, 
Patient Characteristics

\begin{tabular}{|c|c|c|c|c|c|c|c|c|c|}
\hline & $\begin{array}{c}\text { ABA } \\
(n=1,250)\end{array}$ & $\begin{array}{c}\text { ADA } \\
(n=4,986)\end{array}$ & $\begin{array}{c}\text { CER } \\
(\mathbf{n}=387)\end{array}$ & $\begin{array}{c}\text { ETN } \\
(\mathrm{n}=5,266)\end{array}$ & $\begin{array}{c}\text { GOL } \\
(n=577)\end{array}$ & $\begin{array}{c}\text { INF } \\
(\mathrm{n}=969)\end{array}$ & $\begin{array}{c}\text { TOC } \\
(n=451)\end{array}$ & $\begin{array}{c}\text { TOF } \\
(\mathrm{n}=889)\end{array}$ & $\begin{array}{l}\text { All Patients } \\
(\mathrm{N}=14,775)\end{array}$ \\
\hline Age, mean years (SD) & $50.3 \quad(9.1)$ & \begin{tabular}{ll|}
49.1 & $(9.7)$ \\
\end{tabular} & $48.9(10.2)$ & $49.1(10.1)$ & $49.5 \quad(9.6)$ & $50.9 \quad(9.6)$ & \begin{tabular}{ll|}
49.5 & $(9.9)$ \\
\end{tabular} & $\begin{array}{ll}51.7 & (8.5) \\
\end{array}$ & $49.5 \quad(9.8)$ \\
\hline Sex, n female (\%) & $1,067(85.4)$ & $3,871(77.6)$ & $328(84.8)$ & $4,194(79.6)$ & $471(81.6)$ & $769(79.4)$ & $383(84.9)$ & $735(82.7)$ & $11,818(80.0)$ \\
\hline \multicolumn{10}{|l|}{ Geographic region, n (\%) } \\
\hline Northeast & $227(18.2)$ & $698(14.0)$ & $55(14.2)$ & $885(16.8)$ & $110(19.1)$ & $163(16.8)$ & $72(16.0)$ & $181(20.4)$ & $2,391 \quad(16.2)$ \\
\hline North central & $221(17.7)$ & $993(19.9)$ & $63(16.3)$ & $1,039(19.7)$ & $75(13.0)$ & $164(16.9)$ & $88(19.5)$ & $171(19.2)$ & $2,814(19.0)$ \\
\hline South & $583(46.6)$ & $2,490(49.9)$ & $202(52.2)$ & $2,304(43.8)$ & $279(48.4)$ & $500(51.6)$ & $198(43.9)$ & $413(46.5)$ & $6,969(47.2)$ \\
\hline West & $185(14.8)$ & $708(14.2)$ & $61(15.8)$ & $868(16.5)$ & $96(16.6)$ & $132(13.6)$ & $87(19.3)$ & $114(12.8)$ & $2,251(15.2)$ \\
\hline Unknown & $34 \quad(2.7)$ & $\begin{array}{ll}97 & (1.9)\end{array}$ & $6 \quad(1.6)$ & $170 \quad(3.2)$ & $17 \quad(2.9)$ & $10 \quad(1.0)$ & $6 \quad(1.3)$ & $10 \quad(1.1)$ & $350 \quad(2.4)$ \\
\hline \multicolumn{10}{|l|}{ Insurance plan, n (\%) } \\
\hline Traditional/indemnity & $60 \quad(4.8)$ & $162 \quad(3.2)$ & $13 \quad(3.4)$ & $184 \quad(3.5)$ & $13 \quad(2.3)$ & $33 \quad(3.4)$ & $19 \quad(4.2)$ & $28 \quad(3.1)$ & $512 \quad(3.5)$ \\
\hline $\mathrm{EPO} / \mathrm{PPO}$ & $819(65.5)$ & $3,236(64.9)$ & $245(63.3)$ & $3,376(64.1)$ & $350(60.7)$ & $634(65.4)$ & $283(62.7)$ & $566(63.7)$ & $9,509(64.4)$ \\
\hline $\mathrm{HMO}$ & $122 \quad(9.8)$ & $407 \quad(8.2)$ & $48(12.4)$ & $536(10.2)$ & $69(12.0)$ & $110(11.4)$ & $51(11.3)$ & $79 \quad(8.9)$ & $1,422 \quad(9.6)$ \\
\hline POS & $71 \quad(5.7)$ & $401 \quad(8.0)$ & $30 \quad(7.8)$ & $365 \quad(6.9)$ & $36 \quad(6.2)$ & $58 \quad(6.0)$ & $27 \quad(6.0)$ & $63 \quad(7.1)$ & $1,051 \quad(7.1)$ \\
\hline POS with capitation & $17 \quad(1.4)$ & $14 \quad(0.3)$ & $3 \quad(0.8)$ & $\begin{array}{ll}25 & (0.5)\end{array}$ & D & $6 \quad(0.6)$ & $\begin{array}{ll}4 & (0.9) \\
\end{array}$ & $1 \quad(0.1)$ & $70 \quad(0.5)$ \\
\hline Other & $131(10.5)$ & $668(13.4)$ & $42(10.9)$ & 719 (13.7) & $101(17.5)$ & 109 (11.2) & $59(13.1)$ & $130(14.6)$ & $1,959(13.3)$ \\
\hline Unknown & $30 \quad(2.4)$ & $98 \quad(2.0)$ & $6 \quad(1.6)$ & $61 \quad(1.2)$ & $8 \quad(1.4)$ & $19 \quad(2.0)$ & $8 \quad(1.8)$ & $22 \quad(2.5)$ & $252 \quad(1.7)$ \\
\hline $\begin{array}{l}\text { Deyo Charlson } \\
\text { Comorbidity Index, } \\
\text { mean score (SD) }\end{array}$ & $1.4 \quad(1.0)$ & $1.3 \quad(0.7)$ & $1.3 \quad(0.7)$ & $1.3(0.8)$ & $1.3(0.8)$ & $1.3 \quad(0.8)$ & $1.4 \quad(0.9)$ & $1.4 \quad(0.8)$ & $1.3 \quad(0.8)$ \\
\hline \multicolumn{10}{|c|}{ Select comorbid conditions, n (\%) } \\
\hline COPD/asthma & $120 \quad(9.6)$ & $318 \quad(6.4)$ & $31 \quad(8.0)$ & $397 \quad(7.5)$ & $39 \quad(6.8)$ & $75 \quad(7.7)$ & $44 \quad(9.8)$ & $79 \quad(8.9)$ & $1,103 \quad(7.5)$ \\
\hline Diabetes & $146(11.7)$ & $468 \quad(9.4)$ & $24 \quad(6.2)$ & $527(10.0)$ & $59(10.2)$ & $109(11.2)$ & $55(12.2)$ & $101(11.4)$ & $1,489(10.1)$ \\
\hline Dyslipidemia & $219(17.5)$ & $804(16.1)$ & $51(13.2)$ & $885(16.8)$ & $81(14.0)$ & $169(17.4)$ & $96(21.3)$ & $191(21.5)$ & $2,496(16.9)$ \\
\hline Hypertension & $322(25.8)$ & $1,227(24.6)$ & $85(22.0)$ & $1,298(24.6)$ & $150(26.0)$ & $252(26.0)$ & $132(29.3)$ & $274(30.8)$ & $3,740(25.3)$ \\
\hline \multicolumn{10}{|c|}{ Baseline cDMARD use, $\mathrm{n}(\%)$} \\
\hline Hydroxychloroquine & $373(29.8)$ & $1,284(25.8)$ & $79(20.4)$ & $1,383(26.3)$ & $119(20.6)$ & $198(20.4)$ & $87(19.3)$ & $182(20.5)$ & $3,705(25.1)$ \\
\hline Leflunomide & $196(15.7)$ & $611(12.3)$ & $33 \quad(8.5)$ & $627(11.9)$ & $73(12.7)$ & $105(10.8)$ & $54(12.0)$ & $150(16.9)$ & $1,849(12.5)$ \\
\hline Methotrexate & 464 (37.1) & $2,904(58.2)$ & $181(46.8)$ & $2,780(52.8)$ & $305(52.9)$ & $556(57.4)$ & $151(33.5)$ & $371(41.7)$ & $7,712(52.2)$ \\
\hline Sulfasalazine & $106 \quad(8.5)$ & $446 \quad(8.9)$ & \begin{tabular}{|ll}
31 & $(8.0)$ \\
\end{tabular} & $\begin{array}{ll}496 & (9.4) \\
\end{array}$ & $42 \quad(7.3)$ & $\begin{array}{ll}64 & (6.6) \\
\end{array}$ & $19 \quad(4.2)$ & $\begin{array}{ll}72 & (8.1) \\
\end{array}$ & $1,276 \quad(8.6)$ \\
\hline
\end{tabular}

claims data do not include any measures of disease activity level or treatment effectiveness. An effectiveness algorithm was developed and validated against disease activity scores using registry data to estimate effectiveness of therapy in patients with RA. ${ }^{5}$ Multiple studies have used this algorithm to evaluate and compare the effectiveness of RA medications $s^{6,7}$ and to estimate the cost of treatment per effectively treated patients. ${ }^{8-11}$ The previous studies were conducted using claims data up to 2011, since that time, several newer targeted immunomodulators (TIMs) to treat RA have become available. The availability of these drugs has provided more mechanisms of action that may encourage greater switching between drug classes, leading to underestimation of rates of effectiveness based on the algorithm.

The objectives of this study were to (a) describe and summarize the effectiveness of TIMs for the treatment of RA and (b) determine the current RA-related health care costs among RA patients treated with TIMs. These updated data should provide useful information regarding the cost per effective treatment for RA for payers to use in decision making.

\section{Methods}

\section{Data Source}

We used administrative claims data from the IBM MarketScan Commercial Claims and Encounters Database. This database contains deidentified information on geographically diverse, commercially insured patients in the United States. Analyses of health care claims data do not meet the definition of human subject research, and institutional review board approval was therefore not required. No identifiable private information or protected health information was provided.

\section{Study Design}

The study period for this retrospective, observational study was July 1, 2012-December 31, 2016. The index date was the first use of a TIM. A 6-month pre-index period (baseline period) 


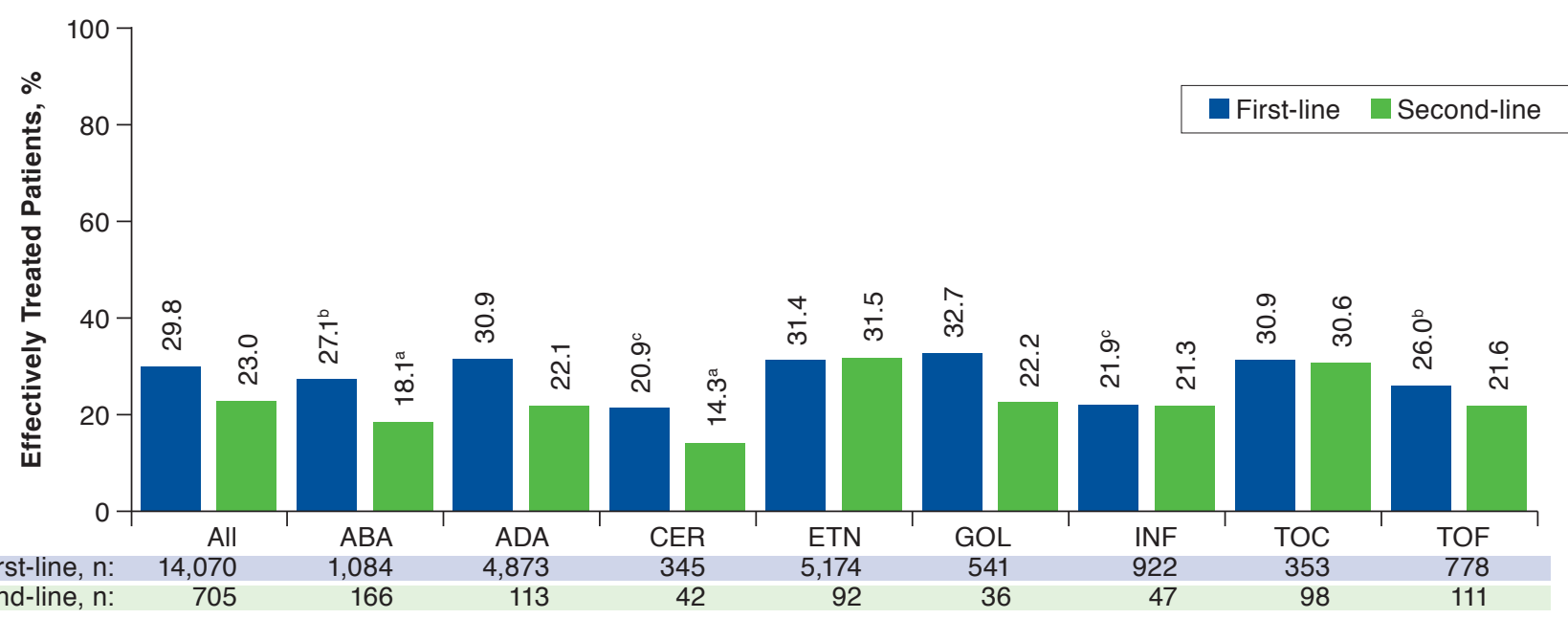

Note: The percentage of patients deemed effectively treated based on the algorithm are shown for patients receiving first-line therapy (blue bars) and second-line therapy (green bars) with TIMs.

aP $<0.05$ vs. ETN.

${ }^{b} P<0.01$ vs. ETN.

${ }^{c} P<0.001$ vs. ETN.

$A B A=$ abatacept $; A D A=$ adalimumab; $C E R=$ certolizumab pegol; $E T N=$ etanercept $; G O L=$ golimumab; INF =infliximab; TIMs = targeted immunomodulators; TOC = tocilizumab; TOF = tofacitini

was used to determine demographic and clinical characteristics, as well as first-line and second-line treatments. Patients were followed for 12 months starting the day after the index date (follow-up period).

TIMs included in this analysis were the following: T-cell costimulation modulator abatacept; the tumor necrosis factor inhibitor (TNFi) medications adalimumab, certolizumab pegol, etanercept, golimumab, and infliximab; the interleukin-6 receptor blocker tocilizumab; and the Janus kinase (JAK) inhibitor tofacitinib.

\section{Patient Identification}

To be eligible, patients had to have an index claim for a TIM between January 1, 2013, and December 31, 2015; had to have continuous enrollment for 6 months before the index date through 12 months after the index date; were aged 18-64 years on the index date; and had $\geq 1$ nondiagnostic medical claim with an International Classification of Diseases, Ninth/Tenth Revision, Clinical Modification (ICD-9-CM or ICD-10-CM) diagnosis code (Appendix A, available in online article) for RA in the primary or secondary position during the baseline period or on the index date. Patients could not have a claim for more than 1 TIM type on the same day that could be considered as an index TIM claim or any inpatient or outpatient nondiagnostic claims in any diagnosis code positions with ICD-9-CM or ICD-10-CM diagnosis codes for any other autoimmune conditions for which TIMs are used during the baseline period through 30 days after the index date (Appendix B, available in online article).

TIM use among patients who had no claims for a TIM of interest during the baseline period was considered first-line therapy. Patients with a claim for a different TIM during the baseline period than the index TIM were considered to be receiving second-line therapy.

\section{Study Outcomes}

Study outcomes included the percentage of patients considered effectively treated for each TIM and RA-related costs per effectively treated patients. Treatment effectiveness was assessed using the validated claims-based effectiveness algorithm. ${ }^{5}$ Costs included total RA-related health care costs (pharmacy and medical excluding administration costs for infusions) for 1 year following index date for effectively treated patients. Health care costs were attributable to RA if there was an RA diagnosis code in any position of a nondiagnostic outpatient medical claim. For hospitalizations, the entire cost was attributable to RA if RA was the primary discharge diagnosis. RA-related medications included conventional disease-modifying antirheumatic drugs (DMARDs) and TIMs. 
TABLE 2 Patients Who Failed Effectiveness Criteria with First-Line TIMs for the Treatment of RA ${ }^{a}$

\begin{tabular}{|c|c|c|c|c|c|c|c|c|c|}
\hline $\begin{array}{l}\text { Patients Who Failed } \\
\text { Criterion, n (\%) }\end{array}$ & $\begin{array}{c}\text { ABA } \\
(n=1,250)\end{array}$ & $\begin{array}{c}\text { ADA } \\
(n=4,986)\end{array}$ & $\begin{array}{c}\text { CER } \\
(\mathrm{n}=387)\end{array}$ & $\begin{array}{c}\text { ETN } \\
(\mathrm{n}=5,266)\end{array}$ & $\begin{array}{c}\text { GOL } \\
(\mathrm{n}=577)\end{array}$ & $\begin{array}{c}\text { INF } \\
(\mathbf{n}=969)\end{array}$ & $\begin{array}{c}\text { TOC } \\
(n=451)\end{array}$ & $\begin{array}{c}\text { TOF } \\
(\mathrm{n}=889)\end{array}$ & $\begin{array}{l}\text { All Patients } \\
(\mathrm{N}=14,775)\end{array}$ \\
\hline Any criterion & $790(72.9)$ & $3,366(69.1)$ & $273(79.1)$ & $3,547(68.6)$ & $364(67.3)$ & $720(78.1)$ & $244(69.1)$ & $576(74.0)$ & $9,880(70.2)$ \\
\hline Nonadherenceb & $688(63.5)$ & $2,824(58.0)$ & $236(68.4)$ & $3,089(59.7)$ & $286(52.9)$ & $388(42.1)$ & $184(52.1)$ & $505(64.9)$ & $8,200(58.3)$ \\
\hline Increased biologic dose & $5 \quad(0.5)$ & $263 \quad(5.4)$ & $8 \quad(2.3)$ & $11 \quad(0.2)$ & $7 \quad(1.3)$ & $354(38.4)$ & $1 \quad(0.3)$ & 0 & $649 \quad(4.6)$ \\
\hline Added new cDMARDc & $97 \quad(8.9)$ & $342 \quad(7.0)$ & $42(12.2)$ & $323 \quad(6.2)$ & $49 \quad(9.1)$ & $78 \quad(8.5)$ & $38(10.8)$ & $51 \quad(6.6)$ & $1,020 \quad(7.2)$ \\
\hline Switched biologicd & $233(21.5)$ & $1,165(23.9)$ & $85(24.6)$ & $1,177(22.7)$ & $138(25.5)$ & $156(16.9)$ & $81(22.9)$ & $147(18.9)$ & $3,182(22.6)$ \\
\hline $\begin{array}{l}\text { Initiated/increased dose } \\
\text { of oral glucocorticoids }\end{array}$ & $214(19.7)$ & $739(15.2)$ & $66(19.1)$ & $660(12.8)$ & $80(14.8)$ & $157(17.0)$ & $64(18.1)$ & $149(19.2)$ & $2,129(15.1)$ \\
\hline $\begin{array}{l}\geq 2 \text { intra-articular } \\
\text { injections }\end{array}$ & $89 \quad(8.2)$ & $342 \quad(7.0)$ & $28(8.1)$ & $364 \quad(7.0)$ & $57(10.5)$ & $66 \quad(7.2)$ & $41(11.6)$ & $67 \quad(8.6)$ & 1,054 \\
\hline
\end{tabular}

a First-line defined as patients without any biologic experience in the baseline period.

${ }^{b}$ For self-administered drugs (abatacept SC, adalimumab, certolizumab, etanercept, golimumab SC, and tocilizumab SC) and tofacitinib, nonadherence was defined as a medication possession ratio of less than $80 \%$. For infused drugs (abatacept IV, golimumab IV and tocilizumab IV), a proportion of days covered was calculated using the expected duration of clinical benefit. For infliximab, nonadherence was defined as not enough infusions corresponding to the lower end of the permissible dosing schedule. 'Defined as the new use of a conventional DMARD (leflunomide, methotrexate, sulfasalazine, or hydroxychloroquine), i.e., patients with no baseline conventional DMARD use or patients who initiate a new conventional DMARD.

dPatient had a claim for a biologic that was different than index biologic during the follow-up period. The biologics used to determine a switch in therapy included abatacept, adalimumab, anakinra, certolizumab, etanercept, golimumab, infliximab, rituximab, tocilizumab, and tofacitinib.

$A B A=$ abatacept $; A D A=$ adalimumab; $c D M A R D=$ conventional disease-modifying antirheumatic drug; $C E R=$ certolizumab pegol; $E T N=$ etanercept; $G O L=$ golimumab; $I N F=$ infliximab; $I V=$ intravenous; $R A=$ rheumatoid arthritis; $S C=$ subcutaneous; TIM = targeted immunomodulator; TOC=tocilizumab; TOF = tofacitinib.

\section{Effectiveness Algorithm}

The algorithm is composed of 6 criteria. Treatment effectiveness was defined as the absence of all of the events listed below during the period from index date through the end of the 12-month follow-up period.

1. Nonadherence. For self-administered drugs (abatacept administered subcutaneously [SC], adalimumab, certolizumab pegol, etanercept, golimumab SC, and tocilizumab SC) and tofacitinib, nonadherence was defined as medication possession ratio (MPR) of $\leq 80 \%$. MPR was calculated as the total days supply for the index therapy divided by the total follow-up days (365 days fixed follow-up period). If a patient refilled a medication before the previous days supply running out, the days supply was extended for a maximum of 14 days (allowing for 14 days of overlap). For infused drugs (abatacept, golimumab, and tocilizumab), a proportion of days covered (PDC) of $\leq 80 \%$ was calculated using the expected duration of clinical benefit. For the infused drug infliximab, nonadherence was defined as too few infusions corresponding to the lower end of the permissible dosing schedule during the follow-up period.

2. Increased index medication dose. The threshold for index medication dose increase was defined separately for each medication. For self-administered drugs (abatacept, adalimumab, certolizumab pegol, etanercept, golimumab, and tocilizumab) and tofacitinib, an increase in index biologic dose was measured by calculating a daily dose for every claim for the index drug during the 12-month follow-up period and comparing that value with the daily dose of the index claim. The patient was categorized as having an increased dose if a subsequent claim had a daily dose greater than the index claim (daily dose $=[$ strength $\times$ quantity $] /$ days supply). For infused drugs (abatacept, golimumab, and tocilizumab), an increase in index biologic dose was measured by calculating the cost per unit of the last claim for the index drug during the 12-month followup period and comparing that value with the cost per unit on the index claim. If the last claim had a cost per unit that was greater than the index claim, the patient was categorized as having a dose increase (cost per unit=paid amount on claim/ wholesale acquisition cost price [matched to year of claim]).

3. Addition of a conventional DMARD. Adding a conventional DMARD (leflunomide, methotrexate, sulfasalazine, or hydroxychloroquine) was defined as the new use of a conventional DMARD (i.e., patients with no baseline conventional DMARD use or patients who initiated a new conventional DMARD).

4. Switch of biologic medications. If a patient had a claim for a biologic that was different than the index drug, they were categorized as having switched treatments. The biologics that were used to determine a switch in therapy included abatacept, adalimumab, anakinra, certolizumab pegol, golimumab, infliximab, rituximab, tocilizumab, and tofacitinib (a JAK inhibitor, although not a biologic, that was included as part of the newer class of specialty drugs for this criterion).

5. Addition of glucocorticoid or increased glucocorticoid dose $\geq 20 \%$ (determined using prednisone dose equivalents). If patients had no prescription for an oral glucocorticoid in the baseline period, they were categorized as adding or initiating an oral glucocorticoid if they had $>30$ days of cumulative oral glucocorticoid use between day 91 after index and the end of the 12-month follow-up period. The dose increase of oral 
TABLE 3 Patients Who Failed Effectiveness Criteria with Second-Line TIMs for the Treatment of RA

\begin{tabular}{|c|c|c|c|c|c|c|c|c|c|}
\hline $\begin{array}{l}\text { Patients Who Failed } \\
\text { Criterion, n (\%) }\end{array}$ & $\begin{array}{c}\text { ABA } \\
(n=166)\end{array}$ & $\begin{array}{c}\text { ADA } \\
(\mathrm{n}=113)\end{array}$ & $\begin{array}{c}\text { CER } \\
(n=42)\end{array}$ & $\begin{array}{c}\text { ETN } \\
(n=92)\end{array}$ & $\begin{array}{c}\text { GOL } \\
(\mathbf{n}=36)\end{array}$ & $\begin{array}{c}\text { INF } \\
(n=47)\end{array}$ & $\begin{array}{c}\text { TOC } \\
(n=98)\end{array}$ & $\begin{array}{c}\text { TOF } \\
(n=111)\end{array}$ & $\begin{array}{l}\text { All Patients } \\
(\mathrm{N}=705)\end{array}$ \\
\hline Any criterion & $136(81.9)$ & $88(77.9)$ & $36(85.7)$ & $63(68.5)$ & $28(77.8)$ & $37(78.7)$ & $68(69.4)$ & $87(78.4)$ & $543(77.0)$ \\
\hline Nonadherence $^{b}$ & $117(70.5)$ & $67(59.3)$ & $34(81.0)$ & $52(56.5)$ & $23(63.9)$ & $21(44.7)$ & $49(50.0)$ & $72(64.9)$ & $435(61.7)$ \\
\hline Increased biologic dose & $2 \quad(1.2)$ & $11 \quad(9.7)$ & $1 \quad(2.4)$ & 0 & 0 & $18(38.3)$ & 0 & 0 & $32 \quad(4.5)$ \\
\hline Added new cDMARDc & $11 \quad(6.6)$ & $10 \quad(8.8)$ & $2 \quad(4.8)$ & $4 \quad(4.3)$ & $9(25.0)$ & $4 \quad(8.5)$ & $7 \quad(7.1)$ & $11 \quad(9.9)$ & $58 \quad(8.2)$ \\
\hline Switched biologicd & $63(38.0)$ & $41(36.3)$ & $15(35.7)$ & $26(28.3)$ & $13(36.1)$ & $15(31.9)$ & $36(36.7)$ & $36(32.4)$ & $245(34.8)$ \\
\hline $\begin{array}{l}\text { Initiated/increased dose } \\
\text { of oral glucocorticoids }\end{array}$ & $39(23.5)$ & $24(21.2)$ & $8(19.0)$ & $13(14.1)$ & $7(19.4)$ & $5(10.6)$ & $21 \quad(21.4)$ & $32(28.8)$ & $149(21.1)$ \\
\hline $\begin{array}{l}\geq 2 \text { intra-articular } \\
\text { injections }\end{array}$ & $17(10.2)$ & $13(11.5)$ & $4 \quad(9.5)$ & $7 \quad(7.6)$ & $5(13.9)$ & $6(12.8)$ & $11(11.2)$ & $15(13.5)$ & 78 (11.1) \\
\hline
\end{tabular}

asecond-line defined as patients with experience of a biologic agent in the baseline period that is different than the index biologic agent.

${ }^{b}$ For self-administered drugs (abatacept SC, adalimumab, certolizumab, etanercept, golimumab SC, and tocilizumab SC) and tofacitinib, nonadherence was defined as a medication possession ratio of less than $80 \%$. For infused drugs (abatacept IV, golimumab IV, and tocilizumab IV), a proportion of days covered was calculated using the expected duration of clinical benefit. For infliximab, nonadherence was defined as not enough infusions corresponding to the lower end of the permissible dosing schedule. 'Defined as the new use of a conventional DMARD (leflunomide, methotrexate, sulfasalazine, or hydroxychloroquine), i.e., patients with no baseline conventional DMARD use or patients who initiate a new conventional DMARD.

dPatient had a claim for a biologic that was different than index biologic during the follow-up period. The biologics used to determine a switch in therapy included abatacept, adalimumab, anakinra, certolizumab, etanercept, golimumab, infliximab, rituximab, tocilizumab, and tofacitinib.

$A B A=$ abatacept $; A D A=$ adalimumab; $c D M A R D=$ conventional disease-modifying antirheumatic drug; $C E R=$ certolizumab pegol; ETN =etanercept; GOL = golimumab; $I N F=$ infliximab; IV=intravenous; $S C=$ subcutaneous; $T I M=$ targeted immunomodulator; $T O C=$ tocilizumab; TOF = tofacitinib.

glucocorticoid was measured in patients who had claims for oral glucocorticoids in the baseline period. Dose increase was defined by capturing the cumulative dose of all glucocorticoid claims during the baseline period and comparing it with the 6 months before the end of the 12-month follow-up period. If the cumulative dose in the second 6 months was $\geq 120 \%$ of the cumulative dose in the baseline period, the patient was categorized as having a dose increase.

6. Receipt of $\geq 2$ intra-articular injections in 1 year. If a patient had $\geq 2$ procedure codes for a joint injection on different days between day 91 after index and the end of the 12-month follow-up period, they were categorized as having excessive parenteral or intra-articular joint injections.

Each criterion was captured independently, so a patient could fail as few as 0 or as many as 6 criteria. Patients were considered effectively treated if they did not meet any of the 6 criteria in the algorithm during the 12-month follow-up period and were considered noneffectively treated if they failed $\geq 1$ of the 6 criteria.

\section{Results}

\section{Patients}

A total of 14,775 patients were eligible to be included in the analysis (Appendix B). The mean age was 49.5 years; most patients were women (80.0\%); and most patients had health care plans with an exclusive provider organization or preferred provider organization (Table 1). The most commonly prescribed TIM was etanercept $(n=5,266)$, followed by adalimumab $(n=4,986)$, abatacept $(n=1,250)$, infliximab $(n=969)$, tofacitinib $(n=889)$, golimumab $(n=577)$, tocilizumab $(n=451)$, and certolizumab pegol $(n=387)$. Few patients $(n=705)$ were receiving secondline therapy during the 12-month follow-up period.

\section{Effectively Treated Patients}

For patients receiving first-line therapy, the golimumab and etanercept cohorts had the highest percentages of effectively treated patients (32.7\% and $31.4 \%$, respectively), followed by adalimumab and tocilizumab (30.9\% each). The certolizumab pegol and infliximab cohorts had the lowest percentages of effectively treated patients $(20.9 \%$ and $21.9 \%$, respectively; Figure 1). Table 2 shows the percentages of patients meeting any of the 6 criteria and the breakdown of each of these criteria by medication cohort among patients receiving first-line therapy. For patients receiving second-line therapy, the etanercept and tocilizumab cohorts had the highest percentages of effectively treated patients (31.5\% and $30.6 \%$, respectively), followed by golimumab (22.2\%) and adalimumab (22.1\%). The certolizumab and abatacept cohorts had the lowest percentages of effectively treated patients ( $14.3 \%$ and $18.1 \%$, respectively; Figure 1). Table 3 shows the percentages of patients failing effectiveness criteria by medication cohort among patients receiving second-line therapy.

The percentage of patients who failed any effectiveness criterion ranged from $67.3 \%$ for golimumab to $79.1 \%$ for certolizumab pegol (Table 2). Patients receiving certolizumab pegol had the highest rates of failing the nonadherence $(68.4 \%)$ and addition of new conventional DMARD criteria (12.2\%); infliximab had the highest rate of increasing biologic dose (38.4\%); golimumab had the highest rate of biologic switching (25.5\%); abatacept had the highest rate of initiating or increasing the 


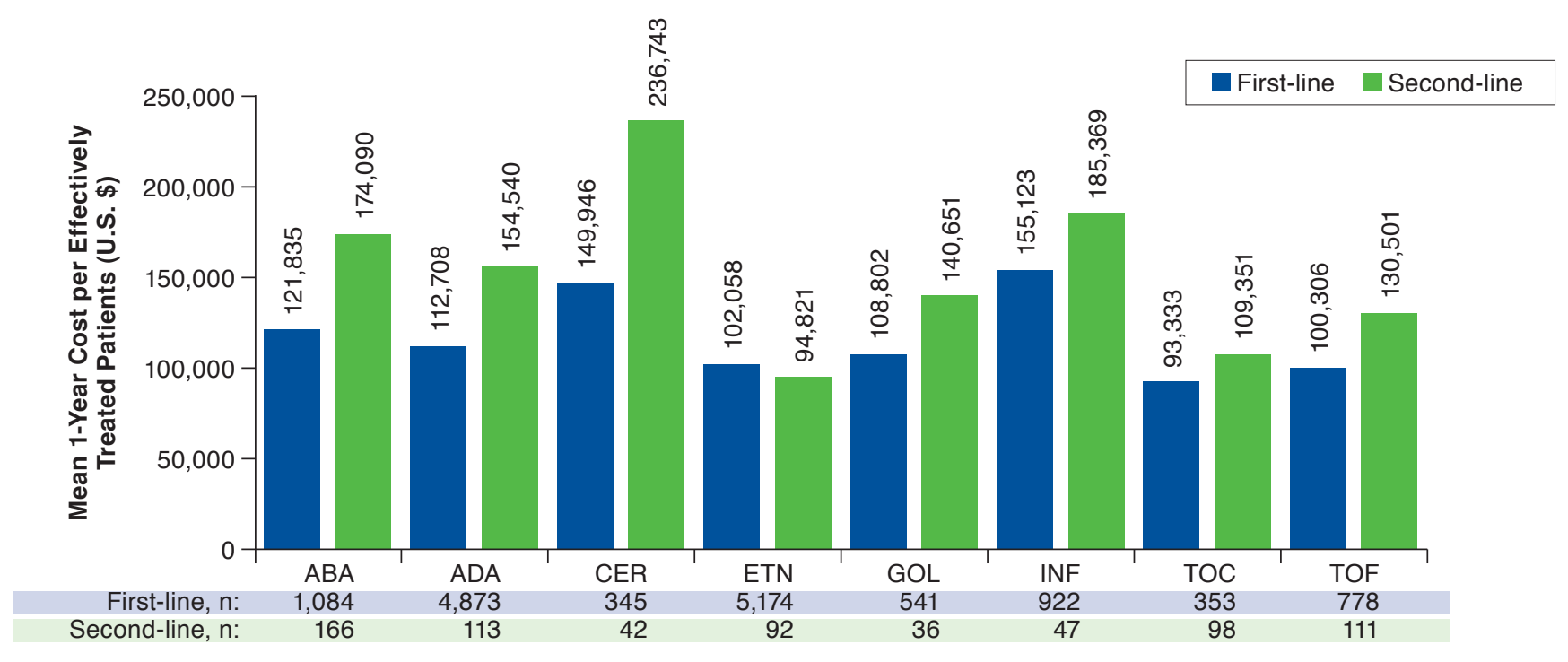

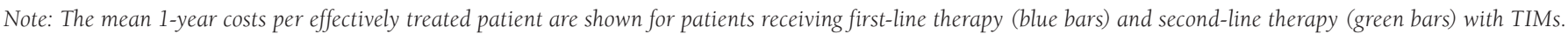
$A B A=$ abatacept $; A D A=$ adalimumab; CER = certolizumab pegol; ETN = etanercept GOL = golimumab; INF=infliximab; TIMs = targeted immunomodulators; TOC $=$ tocilizumab; TOF $=$ tofacitinib.

dose of oral glucocorticoids (19.7\%); and tocilizumab had the highest rate of intra-articular injections (11.6\%). Etanercept had the lowest rates of increased biologic dose $(0.2 \%)$, addition of new cDMARD (6.2\%), initiating or increasing dose of oral glucocorticoids (12.8\%), and receipt of intra-articular injections (7.0\%). Similar patterns were observed for second-line therapies (Table 3).

\section{Cost Per Effectively Treated Patient}

For patients receiving first-line therapy, the tocilizumab and tofacitinib cohorts had the lowest 1-year total RA-related health care cost per effectively treated patient $(\$ 93,333$ and $\$ 100,306$, respectively), followed by etanercept $(\$ 102,058)$ and golimumab $(\$ 108,802$; Figure 2). Except for the etanercept cohort, the cost per effectively treated patient for second-line therapy seemed to be higher than first-line therapy. For patients receiving secondline therapy, the etanercept and tocilizumab cohorts had the lowest 1-year total RA-related health care cost per effectively treated patient $(\$ 94,821$ and $\$ 109,351$, respectively), followed by tofacitinib $(\$ 130,501)$ and golimumab $(\$ 140,651$; Figure 2$)$.

\section{Discussion}

This study evaluated the effectiveness and cost of per effectively treated patient for established RA medications (abatacept, adalimumab, certolizumab pegol, etanercept, golimumab, and infliximab) and new RA medications (tocilizumab and tofacitinib). We found that the golimumab, etanercept, adalimumab, and tocilizumab cohorts seemed to have the highest percentages of effectively treated patients for first-line therapy, and etanercept and tocilizumab cohorts were highest for secondline therapies based on the algorithm. One-year total mean RA-related costs per effectively treated patient were variable, and except for etanercept, costs per effectively treated patients for second-line therapy were higher than costs per effectively treated patients for first-line therapy for each medication.

Rates of effective treatment in our study were similar to those reported in previous studies. ${ }^{8-11}$ This update to previous studies showed that effectiveness of these RA medications has not changed, suggesting that the older medications retain their effectiveness and that the availability of medications with new mechanisms of action has not hastened the need to switch therapy. For those patients who fail effectiveness criteria, older medications and newer medications offer effective alternative options. Effectiveness of second-line therapies for RA has not been extensively studied; however, it is important to understand if medications maintain effectiveness as second-line therapies in real-world settings. Similar to results from another study, ${ }^{12}$ etanercept showed greater effectiveness than other biologic medications as second-line therapy. In our study, except for etanercept, the percentage of effectively treated patients was lower when TIMs were used as second-line therapy compared with their use as first-line therapy. A study by Popp et al. (2018) pointed out that for those medications that have dose adjustment in their labeling, such as infliximab, the removal 
of the "no dose-escalation" criterion drastically reduced the cost per effectively treated patient. ${ }^{13}$ However, it is important to consider that the original validation of the algorithm was only done with these 6 criteria. Therefore, it is unclear how the elimination of 1 criterion may affect the measured clinical effectiveness of these medications.

Switching RA therapy is common in clinical practice. Only 1 in 3 patients in our analysis received effective treatment with their first-line therapy in the first year based on the algorithm, which means many patients received treatment that was not effective over the first year. Studies have shown that failure to respond to an RA therapy is associated with higher costs, including all-cause and RA-related costs. ${ }^{13-15}$ Consistent with those studies, our study showed that, except for etanercept, second-line therapy was associated with higher 1-year total RA-related costs. Recent studies have shown that patients who switch to a TIM with a different mechanism of action than the first-line TIM are more likely to be effectively treated and have generally lower costs with second-line therapy than switching to a TIM with a similar mechanism of action. ${ }^{16-18}$ Future studies are warranted to examine the effectiveness and cost per effectively treated patient for cohorts that switch from one TIM to another with the same mechanism of action compared with those that switch to a TIM with a different mechanism of action.

\section{Limitations}

This study was subject to the usual limitations of a claims-based analysis, including the potential for miscoding. The patient population consisted of individuals with commercial health coverage or private Medicare supplemental coverage; therefore, the results might not be generalizable to RA patients with other types of insurance or without health insurance coverage. Since claims data do not contain disease severity indicators, our analysis could not entirely discard selection bias from the results. Costs did not include rebates or other cost offsets. Our study looked at effectiveness using a claims-based algorithm that was validated for first-line medication use only; therefore, the validity of our effectiveness and cost per effectively treated results for second-line patients may be limited. The population of patients receiving second-line therapy was small, and results should be interpreted with caution.

\section{Conclusions}

This updated analysis of administrative claims data showed that the range of effectiveness of first-line TIMs in real-world practice was higher for certain TNFis and tocilizumab. In contrast to the other TIMs, etanercept, infliximab, and tocilizumab had a similar percentage of effectively treated patients for first-line and second-line therapies, whereas other TIMs had lower rates for second-line therapy. Similarly, only etanercept demonstrated lower 1-year mean total RA-related costs per effectively treated patient, with second-line therapy compared with first-line therapy. The results from this study should assist payers with evaluating effectiveness and costs of currently used TIMs for RA.

\section{Authors}

MAHDI GHARAIBEH, PharmD, MSc, PhD; ERVANT J MAKSABEDIAN HERNANDEZ, PhD, MPhil; and BRADLEY S. STOLSHEK, PharmD, Amgen, Thousand Oaks, California. MACHAON BONAFEDE, PhD, MPH, and DONNA MCMORROW, BS, IBM Watson Health, Cambridge, Massachusetts.

AUTHOR CORRESPONDENCE: Ervant J. Maksabedian Hernandez, PhD, MPhil, Amgen, One Amgen Center Dr., Thousand Oaks, CA 91320.Tel.: 805.447.8979; Email: emaksabe@amgen.com.

\section{DISCLOSURES}

This study was sponsored by Amgen. Amgen employees contributed to study design, analysis of the data, and the decision to publish the results. Maksabedian Hernandez and Stolshek are employees and shareholders of Amgen; Gharaibeh was employed by Amgen at the time of this study. Bonafede was employed by IBM Watson Health, at the time of this study, and McMorrow is employed by IBM Watson Health, which received funding from Amgen to conduct this study.

Data from this study were presented at AMCP Nexus, October 22-25, 2018, in Orlando, FL.

\section{ACKNOWLEDGMENTS}

Dikran Toroser (Amgen) and Julia R. Gage (on behalf of Amgen) provided assistance with writing the manuscript.

\section{DATA SHARING}

Qualified researchers may request data from Amgen clinical studies Complete details are available at the following: https://www.ext.amgen.com/ science/clinical-trials/clinical-data-transparency-practices/.

\section{REFERENCES}

1. Myasoedova E, Crowson CS, Kremers HM, Therneau TM, Gabriel SE. Is the incidence of rheumatoid arthritis rising? Results from Olmsted County, Minnesota, 1955-2007. Arthritis Rheum. 2010;62(6):1576-82.

2. Ma VY, Chan L, Carruthers KJ. Incidence, prevalence, costs, and impact on disability of common conditions requiring rehabilitation in the United States: stroke, spinal cord injury, traumatic brain injury, multiple sclerosis, osteoarthritis, rheumatoid arthritis, limb loss, and back pain. Arch Phys Med Rehabil. 2014;95(5):986-95.el.

3. Fazal SA, Khan M, Nishi SE, et al. A clinical update and global economic burden of rheumatoid arthritis. Endocr Metab Immune Disord Drug Targets. 2018;18(2):98-109.

4. Centers for Disease Control and Prevention. National and state medical expenditures and lost earnings attributable to arthritis and other rheumatic conditions-United States, 2003. MMWR Morb Mortal Wkly Rep. 2007;56(1):4-7.

5. Curtis JR, Baddley JW, Yang S, et al. Derivation and preliminary validation of an administrative claims-based algorithm for the effectiveness of medications for rheumatoid arthritis. Arthritis Res Ther. 2011;13(5):R155. 
6. Oladapo A, Barner JC, Lawson KA, et al. Medication effectiveness with the use of tumor necrosis factor inhibitors among Texas Medicaid patients diagnosed with rheumatoid arthritis. J Manag Care Spec Pharm. 2014;20(7):657-67. Available at: https://www.jmcp.org/doi/10.18553/ jmcp.2014.20.7.657.

7. Yun H, Xie F, Delzell E, et al. The comparative effectiveness of biologics among older adults and disabled rheumatoid arthritis patients in the Medicare population. Br J Clin Pharmacol. 2015;80(6):1447-57.

8. Curtis JR, Schabert VF, Yeaw J, et al. Use of a validated algorithm to estimate the annual cost of effective biologic treatment for rheumatoid arthritis. J Med Econ. 2014;17(8):555-66.

9. Curtis JR, Schabert VF, Harrison DJ, et al. Estimating effectiveness and cost of biologics for rheumatoid arthritis: application of a validated algorithm to commercial insurance claims. Clin Ther. 2014;36(7):996-1004.

10. Curtis JR, Chastek B, Becker L, et al. Cost and effectiveness of biologics for rheumatoid arthritis in a commercially insured population. J Manag Care Spec Pharm. 2015;21(4):318-29. Available at: https://www.jmcp.org/ doi/10.18553/jmcp.2015.21.4.318.

11. Wu N, Bhurke S, Shah N, Harrison DJ. Application of a validated algorithm to estimate the effectiveness and cost of biologics for rheumatoid arthritis in the US pharmacy benefit manager context. Clinicoecon Outcomes Res. 2015;7:257-66.

12. Cantini F, Niccoli L, Nannini C, et al. Second-line biologic therapy optimization in rheumatoid arthritis, psoriatic arthritis, and ankylosing spondylitis. Semin Arthritis Rheum. 2017;47(2):183-92.
13. Popp RA, Rascati K, Davis M, Patel U . Refining a claims-based algorithm to estimate biologic medication effectiveness and cost per effectively treated patient with rheumatoid arthritis. Pharmacotherapy. 2018;38(2):172-80.

14. Grabner M, Boytsov NN, Huang Q, Zhang X, Yan T, Curtis JR. Costs associated with failure to respond to treatment among patients with rheumatoid arthritis initiating TNFi therapy: a retrospective claims analysis. Arthritis Res Ther. 2017;19(1):92.

15. Strand V, Tundia N, Song Y, Macaulay D, Fuldeore M. Economic burden of patients with inadequate response to targeted immunomodulators for rheumatoid arthritis. J Manag Care Spec Pharm. 2018;24(4):344-52. Available at: https://www.jmcp.org/doi/10.18553/jmcp.2018.24.4.344.

16. Bonafede MM, Curtis JR, McMorrow D, Mahajan P, Chen CI. Treatment effectiveness and treatment patterns among rheumatoid arthritis patients after switching from a tumor necrosis factor inhibitor to another medication. Clinicoecon Outcomes Res. 2016;8:707-15.

17. Chastek B, Becker LK, Chen CI, Mahajan P, Curtis JR. Outcomes of tumor necrosis factor inhibitor cycling versus switching to a diseasemodifying anti-rheumatic drug with a new mechanism of action among patients with rheumatoid arthritis. J Med Econ. 2017;20(5):464-73.

18. Bonafede MMK, McMorrow D, Proudfoot C, Shinde S, Kuznik A, Chen CI. Treatment persistence and healthcare costs among patients with rheumatoid arthritis after a change in targeted therapy. Am Health Drug Benefits. 2018;11(4):192-202. 


\begin{tabular}{|c|c|c|}
\hline Code Type & Codes & Condition \\
\hline$\overline{\text { ICD-9 DX }}$ & 7140 & $\begin{array}{l}\text { Rheumatoid } \\
\text { arthritis }\end{array}$ \\
\hline ICD-9 DX & 7200 & $\begin{array}{l}\text { Ankylosing } \\
\text { spondylitis }\end{array}$ \\
\hline ICD-9 DX & $5550-5552,5559$ & Crohn's disease \\
\hline ICD-9 DX & $20410-20412$ & $\begin{array}{l}\text { Chronic } \\
\text { lymphocytic } \\
\text { leukemia }\end{array}$ \\
\hline ICD-9 DX & $71430-71433$ & $\begin{array}{l}\text { Juvenile idiopathic } \\
\text { arthritis }\end{array}$ \\
\hline ICD-9 DX & $\begin{array}{l}20000-20018,20020-20028,20030-20038,20040-20048,20050-20058,20060-20068,20070-20078, \\
20080-20088,20200-20208,20210-20218,20220-20228,20230-20238,20240-20248,20250-20258, \\
20260-20268,20270-20278,20280-20288,20290-20298\end{array}$ & $\begin{array}{l}\text { Non-Hodgkin's } \\
\text { lymphoma }\end{array}$ \\
\hline ICD-9 DX & 6960 & Psoriatic arthritis \\
\hline ICD-9 DX & 6961 & Plaque psoriasis \\
\hline ICD-9 DX & $5560-5566,5568,5569$ & Ulcerative colitis \\
\hline ICD-9 DX & 4464 & $\begin{array}{l}\text { Wegener's } \\
\text { granulomatosis }\end{array}$ \\
\hline ICD-9 DX & 46611,46619 & Bronchiolitis \\
\hline ICD-9 DX & 49300-49302, 49310-49312, 49320-49322, 49390-49392, 496, 49121-49122, 49381, 49382 & $\begin{array}{l}\text { Chronic obstructive } \\
\text { pulmonary disease }\end{array}$ \\
\hline ICD-9 DX & $\begin{array}{l}25000-25003,25010-25013,25020-25023,25030-25033,25040-25043,25050-25053,25060-25063, \\
25070-25073,25080-25083,25090-25093\end{array}$ & Diabetes mellitus \\
\hline ICD-9 DX & (2: & Dyslipidemia \\
\hline ICD-9 DX & $\begin{array}{l}4010,4011,4019,40200,40201,40210,40211,40290,40291,40300,40301,40390,40391,40400- \\
40403,40410-40413,40490-40493,40501,40509,40511,40519,40591,40599\end{array}$ & Hypertension \\
\hline ICD-9 DX & 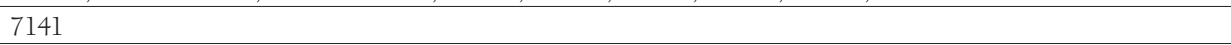 & Felty's syndrome \\
\hline ICD-9 DX & 71481 & Rheumatoid lung \\
\hline ICD-9 DX & 7822 & $\begin{array}{l}\text { Rheumatoid } \\
\text { nodules }\end{array}$ \\
\hline ICD-9 DX & 7102 & Sjögren's syndrome \\
\hline ICD-9 DX & $3541-3544,3548-3550,3552-3554,35571,35579,3558-3559,36218,4465-4467,4476$ & Vasculitis \\
\hline ICD-10 DX & $\begin{array}{l}\text { M0540, M05411-M05412, M05419, M05421-M05422, M05429, M05431-M05432, M05439, } \\
\text { M05441-M05442, M05449, M05451, M05452, M05459, M05461-M05462, M05469, M05471-M05472, } \\
\text { M05479, M0549, M0550, M05511, M05512, M05519, M05521, M05529, M05531, M05532, M05539, } \\
\text { M05541, M05542, M05549, M05551, M05522, M05559, M05561, M05562, M05569, M05571, M05572, } \\
\text { M05579, M0559, M0570, M05711, M05712, M05719, M05721, M05722, M05729, M05731, M05732, } \\
\text { M05739, M05741, M05742, M05749, M05751, M05752, M5759, M05761, M05762, M05769, M05771, } \\
\text { M05772, M05779, M0579, M0580, M05811, M05812, M05819, M05821, M05822, M05829, M05831, } \\
\text { M05832, M05839, M05841, M05842, M05849, M05851, M05852, M05859, M05861, M05862, M05869, } \\
\text { M05871, M05872, M05879, M0589, M059, M0600, M06011, M06012, M06019, M06021, M06022, } \\
\text { M06029, M06031, M06032, M06039, M06041, M06042, M06049, M06051, M06052, M06059, M06061, } \\
\text { M06062, M06069, M06071, M06072, M06079, M0608, M0609, M0620, M06211, M06212, M06219, } \\
\text { M06221, M06222, M06229, M06231, M06232, M06239, M06241, M06242, M06249, M06251, M06252, } \\
\text { M06259, M06261, M06262, M06269, M06271, M06272, M06279, M0628, M0629, M0680, M06811, } \\
\text { M06812, M06819-M06822, M06829, M06831, M06832, M06839, M06841, M06842, M06849, M06851, } \\
\text { M06852, M06859, M06861, M06862, M06869, M06871, M06872, M06879, M0688, M0689, M069 }\end{array}$ & $\begin{array}{l}\text { Rheumatoid } \\
\text { arthritis }\end{array}$ \\
\hline ICD-10 DX & M081, M450-M459, M488X1-M488X9 & $\begin{array}{l}\text { Ankylosing } \\
\text { spondylitis }\end{array}$ \\
\hline ICD-10 DX & $\begin{array}{l}\text { K5000, K50011-K50014, K50018, K50019, K5010, K50111-K50114, K50118, K50119, K50811-K50814, } \\
\text { K5090, K50911-K50914, K50918, K50919 }\end{array}$ & Crohn's disease \\
\hline ICD-10 DX & C9110-C9112 & $\begin{array}{l}\text { Chronic } \\
\text { lymphocytic } \\
\text { leukemia }\end{array}$ \\
\hline
\end{tabular}




\section{APPENDIX A ICD-9-CM and ICD-10-CM Codes (continued)}

Code Type

ICD-10 DX

\section{Codes}

M0800, M08011, M08012, M08019, M08021, M08022, M08029, M08031, M08032, M08039, M08041 M08042, M08049, M08051, M08052, M08059, M08061, M08062, M08069, M08071, M08072, M08079, M0808, M0809, M0820, M08211, M08212, M08219, M08221, M08222, M08229, M08231, M08232. M08239, M08241, M08242, M08249, M08251, M08252, M08259, M08261, M08262, M08269, M08271, M08272, M08279, M0828, M0829, M083, M0840, M080411, M08412, M08419, M08421, M08422, M08429, M08431, M08432, M08439, M08441, M08442, M08449, M08451, M08452, M08459, M08461, M08462, M08469, M08471, M08472, M08479, M0848, M0880, M08811, M08812, M08819, M08821, M08822, M08829, M08831, M08832, M08839, M08841, M08842, M08849, M08851, M08852, M08859, M08861, M08862, M08869, M08871, M08872, M08879, M0888, M0889, M0890, M08911, M08912, M08919, M08921, M08922, M08929, M08931, M08932, M08939, M08941, M08942, M08949, M08951, M08952, M08959, M08961, M08962, M08969, M08971, M08972, M08979, M0898, M0899

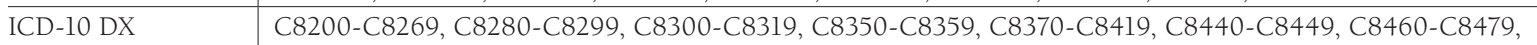
C8490-C8499, C84A0-C84A9, C84Z0-C84Z9, C8510-C8529, C8580-C8599, C860-C866, C880, C883, C884, C96A

ICD-10 DX

ICD-10 DX

ICD-10 DX L4050-L4054, L4059

L400

K5100, K51011-K51014, K51018, K51019, K5120, K51211-K51214, K51218, K51219, K5130,

K51311-K51314, K51318, K51319, K5140, K51411-K51414, K51418, K51419, K5150, K51511-K51514, K51518, K51519, K5180, K51811-K51814, K51818, K51819, K5190, K51911-K51914, K51918, K51919

\begin{tabular}{l|l}
\hline ICD-10 DX & J210, J211, J218, J219 \\
\hline ICD-10 DX & J430-J432, J438-J441, J449, J4520- J4522, J4530-J4532, J4540-J4542, J4550-J4552, J45901, J45902, J45909,
\end{tabular}
J45990, J45991. J45998, J982, J983

ICD-10 DX

E1010, E1011, E1021, E1022, E1029, E10311, E10319, E10321, E10329, E10331, E10339, E10341, E10349, E10351, E10359, E1036, E1039-E1044, E1049, E1051, E1052, E1059, E10610, E10618, E10620-E10622, E10628, E10630, E10638, E10641, E10649, E1065, E1069, E108, E109, E1100, E1101, E1121, E1122, E1129, E11311, E11319, E11321, E11329, E11331, E11339, E11341, E11349, E11351, E11359, E1136, E1139-E1144, E1149, E1151, E1152, El159, E11610, El1618, El1620-E11622, E11628, E11630, E11638, E11641, E11649, E1165, E1169, E118, E119, E1300, E1301, E1310, E1311, E1321, E1322, E1329, E13311, E13319, E13321, E13329, E13331, E13339, E13341, E13349, E13351, E13359, E1336, El339-E1344, E1349, E1351, E1352, E1359, E13610, E13618, E13620-E13622, E13628, E13630, E13638, E13641, E13649, E1365, E1369, E138, E139

\begin{tabular}{|c|c|c|}
\hline ICD-10 DX & E780-E786 & Dyslipidemia \\
\hline ICD-10 DX & I10, I110, I119, I120, I129, I130, I1311, I132 & Hypertension \\
\hline ICD-10 DX & $\begin{array}{l}\text { M0500, M05011, M05012, M05019, M05021, M05022, M05029, M05031, M05032, M05039, M05041, } \\
\text { M05042, M05049, M05051, M05052, M05059, M05061, M05062, M05069, M05071, M05072, M05079, } \\
\text { M0509 }\end{array}$ & Felty's syndrome \\
\hline ICD-10 DX & $\begin{array}{l}\text { M0510, M05111, M05112, M05119, M05121, M05122, M05129, M05131, M05132, M05139, M05141, } \\
\text { M05142, M05149, M05151, M05152, M05159, M05161, M05162, M05169, M05171, M05172, M05179, } \\
\text { M0519 }\end{array}$ & Rheumatoid lung \\
\hline ICD-10 DX & $\begin{array}{l}\text { M0630, M06311, M06312, M06319, M06321, M06322, M06329, M06331, M06332, M06339, M06341, } \\
\text { M06342, M06349, M06351, M06352, M06359, M06361, M06362, M06369, M06371, M06372, M06379, } \\
\text { M0638, M0639 }\end{array}$ & $\begin{array}{l}\text { Rheumatoid } \\
\text { nodules }\end{array}$ \\
\hline ICD-10 DX & M3500-M3504, M3509 & Sjögren's syndrome \\
\hline ICD-10 DX & M3130, M3131 & $\begin{array}{l}\text { Wegener's } \\
\text { granulomatosis }\end{array}$ \\
\hline ICD-10 DX & $\begin{array}{l}\text { M301-M303, M308, M310-M312, M314-M316, G5600-G5602, G5610-G5612, G5620-G5622, } \\
\text { G5630-G5632, G5640-G5642, G5680-G5682, G5690-G5692, H35061-H35063, H35069 }\end{array}$ & Vasculitis \\
\hline
\end{tabular}




\section{APPENDIX B Patient Identification}

Inclusion Criteria

Patients in the MarketScan Commercial Database with a claim for selected TIM (abatacept, adalimumab, certolizumab pegol, etanercept, golimumab, infliximab, tocilizumab, or tofacitinib) between January 2, 2013, and December 31, 2016 (date of first claim was index date)

Did not have $>1$ TIM type on the same day that could be considered as an index TIM claim

Had $\geq 6$ months continuous enrollment and pharmacy benefits before index date (baseline period)

$\mathrm{Had} \geq 12$ months continuous enrollments and pharmacy benefits after index date (follow-up period)

Was aged 18-64 years at index date

$\mathrm{Had} \geq 1$ nondiagnostic medical claim with an ICD-9-CM or ICD-10-CM diagnosis code for RA in the primary or secondary position during the baseline period or on index date

Did not have any inpatient or outpatient nondiagnostic claims in any diagnosis code position with ICD-9-CM or ICD-10-CM

diagnosis codes for any other autoimmune conditions for which biologic TIMs are used during the baseline period and up to 30 days after index date

Did not have data quality issues related to treatment patterns or dosing with index TIM claims

Final sample

Remaining N

160,396

CD-9/10-CM = International Classification of Diseases, Ninth/Tenth Revision, Clinical Modification; $R A=$ rheumatoid arthritis; TIMs=targeted immunomodulators. 